\title{
Electronic Properties of InGaN/GaN Vertical-cavity Lasers
}

Joachim Piprek, Zhan-Ming Li, Robert Farrell, Steven P. DenBaars, and Shuji Nakamura

19.1

Introduction to Vertical-cavity Lasers

In vertical-cavity surface-emitting lasers (VCSELs), the optical cavity is formed by mirrors above and below the gain region (Fig. 19.1(a)). The laser light propagates in a vertical direction and typically exhibits a circular beam shape. Internally, the photons pass the gain region in a perpendicular direction, i.e., optical gain is provided over a short propagation distance only and the amplification per photon round trip is small. Therefore, the mirrors need to be highly reflective so that photons make many round trips before they are emitted. To achieve high reflectivity, distributed Bragg reflectors (DBRs) are used with two alternating layers of high refractive index contrast. With quarter-wavelength layer thickness, the reflected waves from all DBR interfaces add up constructively, allowing for DBR reflectivities above 99\% [1].

Within the active layers (quantum wells), optical gain arises from the stimulated recombination of electrons and holes, which may be generated by optical absorption of pump light or by current injection. The latter is more difficult to accomplish and it is strongly affected by the electrical DBR properties. Semiconductor DBRs allow for vertical carrier injection through the DBR directly into the active gain region (Fig. 19.1(b)) which gives good overlap of the lateral carrier and photon profile. However, suitable semiconductor materials often exhibit small index contrast and the many hetero-interfaces tend to generate a high electrical DBR resistance. An alternative choice is dielectric DBRs which typically provide a large refractive index contrast so that a few layer pairs are often sufficient for high mirror reflectivity. However, dielectric DBRs are electrically insulating and the injection current needs to be funneled into the active region from the side, typically by using ring contacts around the DBR (Fig. 19.1(c)). Some type of electrical confinement structure is required that forces the carriers to move into the small center region where the optical mode is located [2]. Last, but not least, the small lateral extension of the active region causes a potentially high thermal resistance so that good thermal 

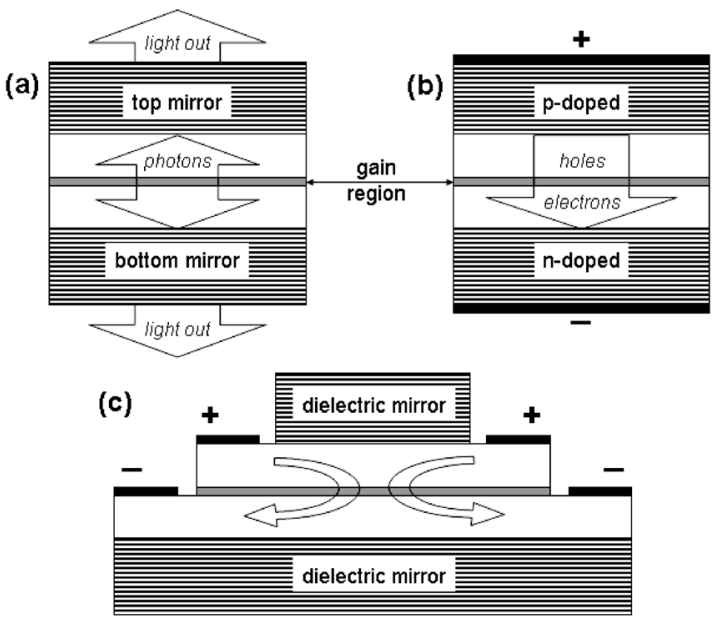

Fig. 19.1 Illustration of vertical-cavity laser principles: (a) vertical photon flux; (b) vertical current flow with semiconductor mirrors; (c) current flow with dielectric mirrors.

conductivity is another essential requirement of VCSEL design [3]. A more detailed review on VCSELs is given in [4].

VCSELs exhibit several advantages over in-plane lasers, including lower manufacturing costs, circular output beams, and longer lifetime [2]. However, the complex interaction of electrical, thermal, and optical processes in VCSELs often requires advanced computer simulation for device analysis and design optimization [5]. Using recently fabricated GaN-VCSELs as an example [6], this chapter demonstrates how advanced simulations are used to analyze internal physics and reveal performance limitations of real devices. Section 19.2 explains the device structure. Section 19.3 describes the VCSEL model and the material parameters used. Simulation results are employed in Section 19.4 to gain deeper insight into internal physical processes.

\section{2}

\section{GaN-based VCSEL Structure}

In contrast to the success of GaAs-based VCSELs in recent years, the demonstration of GaN-based VCSELs still faces significant challenges [7]. Currentinjected GaN-VCSELs often suffer from high optical loss due to insufficient reflectance of semiconductor DBRs, low conductivity of p-GaN, carrier leakage, or the resistance of $\mathrm{GaN}$ to conventional wet etching. Some of these problems have been addressed by the device design presented in [6] (Fig. 19.2, Table 19.1). Eleven-period dielectric DBRs are used on both sides which ex- 


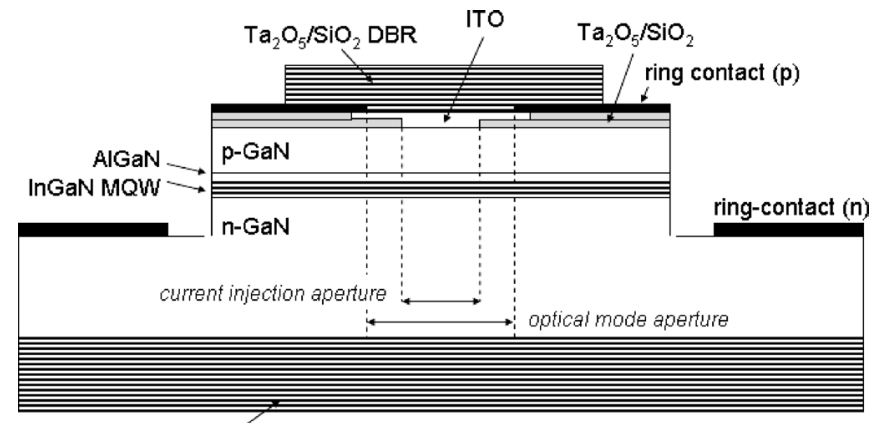

$\mathrm{Ta}_{2} \mathrm{O}_{5} / \mathrm{SiO}_{2} \mathrm{DBR}$

Fig. 19.2 Schematic structure of the vertical-cavity laser investigated.

hibit a high reflectance above $99 \%$. The multi-quantum well (MQW) active region consists of five $4 \mathrm{~nm}$ thick $\mathrm{In}_{0.1} \mathrm{Ga}_{0.9} \mathrm{~N}$ quantum wells and $8 \mathrm{~nm}$ wide $\mathrm{In}_{0.035} \mathrm{Ga}_{0.965} \mathrm{~N}$ barriers. It is covered by a $20 \mathrm{~nm}$ p-doped $\mathrm{Al}_{0.18} \mathrm{Ga}_{0.82} \mathrm{~N}$ electron stopper layer to reduce electron leakage into the $\mathrm{p}-\mathrm{GaN}$ spacer layer. Indium tin oxide (ITO) is employed for lateral leveling of the injection current. However, due to common difficulties with thinning GaN in a well-controlled fashion, the cavity length is as large as $5.5 \mu \mathrm{m}$. The measured longitudinal mode spacing is correspondingly small $(4-5 \mathrm{~nm})$ which eliminates the usual VCSEL design challenge of aligning gain peak and cavity mode [8]. The dense mode spectrum promises a reduced temperature sensitivity of the threshold current.

\section{3}

Theoretical Models and Material Parameters

Due to the complexity of VCSEL physics, realistic device simulations often need to include models for electronic, optical, and thermal processes [9]. In this chapter we focus on electronic processes at room temperature considering pulsed laser operation without significant self-heating. We employ the simulation software PICS3D [10] which self-consistently combines the computation of carrier transport, electron band structure, optical gain, and optical mode. Each model is described below. A similar approach was previously used to study InGaN/GaN in-plane lasers, resulting in excellent agreement between measurement and simulation [11].

An important issue in any device simulation is the selection of appropriate values for the various material parameters. Published parameter values for GaN-based compounds often vary substantially (see, e.g., the review of band structure parameters in [12]). We mainly employ the parameters used successfully in our previous investigations, most of which are listed in Table 19.1 and Table 19.3. Crucial parameters are discussed below. 
Tab. 19.1 VCSEL layer structure and material parameters ( $t$ - layer thickness, $N$ - carrier concentration from doping, $n_{\mathrm{r}}$ - refractive index).

\begin{tabular}{|c|c|c|c|}
\hline Parameter & $t$ & $N$ & $n_{\mathrm{r}}$ \\
\hline Unit & $\mathrm{nm}$ & $\mathrm{cm}^{-3}$ & \\
\hline $\mathrm{SiO}_{2}(11 \times$ in top $\mathrm{DBR})$ & 70 & - & 1.47 \\
\hline $\mathrm{Ta}_{2} \mathrm{O}_{5}(11 \times$ in top DBR $)$ & 47 & - & 2.2 \\
\hline ITO (contact) & 40 & $10 . \times 10^{18}$ & 2.1 \\
\hline p-GaN (spacer) & 540 & $0.4 \times 10^{18}$ & 2.55 \\
\hline $\mathrm{p}-\mathrm{Al}_{0.18} \mathrm{Ga}_{0.82} \mathrm{~N}$ (stopper) & 20 & $10^{16}-10^{18}$ & 2.27 \\
\hline $\mathrm{i}-\ln _{0.035} \mathrm{Ga}_{0.965} \mathrm{~N}$ (barrier) & 8 & - & 2.68 \\
\hline i- $\ln _{0.1} \mathrm{Ga}_{0.9} \mathrm{~N}$ (quantum well) & 4 & - & 3.0 \\
\hline $\mathrm{n}-\ln _{0.035} \mathrm{Ga}_{0.965} \mathrm{~N}$ (barrier) & 8 & $1.0 \times 10^{18}$ & 2.68 \\
\hline i- $\ln _{0.1} \mathrm{Ga}_{0.9} \mathrm{~N}$ (quantum well) & 4 & - & 3.0 \\
\hline $\mathrm{n}-\ln _{0.035} \mathrm{Ga}_{0.965} \mathrm{~N}$ (barrier) & 8 & $1.0 \times 10^{18}$ & 2.68 \\
\hline i- $\ln _{0.1} \mathrm{Ga}_{0.9} \mathrm{~N}$ (quantum well) & 4 & - & 3.0 \\
\hline $\mathrm{n}-\ln _{0.035} \mathrm{Ga}_{0.965} \mathrm{~N}$ (barrier) & 8 & $1.0 \times 10^{18}$ & 2.68 \\
\hline $\mathrm{i}-\mathrm{In}_{0.1} \mathrm{Ga}_{0.9} \mathrm{~N}$ (quantum well) & 4 & - & 3.0 \\
\hline $\mathrm{n}-\mathrm{In}_{0.035} \mathrm{Ga}_{0.965} \mathrm{~N}$ (barrier) & 8 & $1.0 \times 10^{18}$ & 2.68 \\
\hline $\mathrm{i}-\mathrm{In}_{0.1} \mathrm{Ga}_{0.9} \mathrm{~N}$ (quantum well) & 4 & - & 3.0 \\
\hline $\mathrm{n}-\ln _{0.035} \mathrm{Ga}_{0.965} \mathrm{~N}$ (barrier) & 8 & $1.0 \times 10^{18}$ & 2.68 \\
\hline n-GaN (spacer) & 5300 & $2.5 \times 10^{18}$ & 2.55 \\
\hline$\overline{\mathrm{SiO}_{2}(11 \times \text { in bottom DBR })}$ & 70 & - & 1.47 \\
\hline $\mathrm{Ta}_{2} \mathrm{O}_{5}(11 \times$ in bottom DBR $)$ & 47 & - & 2.2 \\
\hline
\end{tabular}

19.3.1

Carrier Transport

PICS3D employs the traditional drift-diffusion model for semiconductors. The current density of electrons $\vec{j}_{n}$ and holes $\vec{j}_{p}$ is caused by the electrostatic field $\vec{F}$ (drift) and by the concentration gradient of electrons and holes, $\nabla n$ and $\nabla p$, respectively,

$$
\begin{aligned}
& \vec{j}_{n}=q \mu_{n} n \vec{F}+q D_{n} \nabla n \\
& \vec{j}_{p}=q \mu_{p} p \vec{F}-q D_{p} \nabla p
\end{aligned}
$$

with the elementary charge $q$, the carrier densities $n$ and $p$, and their mobilities $\mu_{n}$ and $\mu_{p}$, respectively. The diffusion constants $D_{n}$ and $D_{p}$ are replaced by mobilities using the Einstein relation $D=\mu k_{\mathrm{B}} T / q$ with the Boltzmann constant $k_{\mathrm{B}}$ and the temperature $T$. The electric field is affected by the charge distribution, which includes electrons $n$ and holes $p$, dopant ions $\left(p_{\mathrm{D}}, n_{\mathrm{A}}\right)$, and other fixed charges $N_{\mathrm{f}}$ that are of special importance in GaN-based devices in order to account for built-in polarization. This relationship is described by the 
Poisson equation

$$
\nabla \cdot\left(\varepsilon \varepsilon_{0} \vec{F}\right)=q\left(p-n+p_{\mathrm{D}}-n_{\mathrm{A}} \pm N_{\mathrm{f}}\right)
$$

with the free-space permittivity $\varepsilon_{0}$. Changes in the local carrier concentration are connected to a spatial change in current flow $\nabla \vec{j}$ or to the local recombination rate $R$ of electron-hole pairs, as described by the continuity equations

$$
\begin{aligned}
& q \frac{\partial n}{\partial t}=\nabla \cdot \vec{j}_{n}-q R \\
& q \frac{\partial p}{\partial t}=-\nabla \cdot \vec{j}_{p}-q R
\end{aligned}
$$

The relevant carrier recombination mechanisms in GaN-based VCSELs are stimulated photon emission, spontaneous photon emission, and nonradiative Shockley-Read-Hall (SRH) recombination. The stimulated emission of photons is the key physical mechanism in lasers and it is described in Section 19.3.4 together with the spontaneous photon emission in quantum wells. Within bulk layers, the local spontaneous emission rate is approximated by

$$
R_{\mathrm{sp}}=B\left(n p-n_{\mathrm{i}}^{2}\right)
$$

using the bimolecular recombination coefficient $B=5 \times 10^{-11} \mathrm{~cm}^{3} \mathrm{~s}^{-1}$ [13] $\left(n_{\mathrm{i}}\right.$ - intrinsic density). The defect-related SRH recombination rate is given by

$$
R_{\mathrm{SRH}}=\frac{n p-n_{\mathrm{i}}^{2}}{\tau_{p}^{\mathrm{SRH}}\left(n+N_{\mathrm{c}} \exp \left[\frac{E_{\mathrm{t}}-E_{\mathrm{c}}}{k_{\mathrm{B}} T}\right]\right)+\tau_{n}^{\mathrm{SRH}}\left(p+N_{\mathrm{v}} \exp \left[\frac{E_{\mathrm{v}}-E_{\mathrm{t}}}{k_{\mathrm{B}} T}\right]\right)}
$$

and it is governed by the SRH lifetimes $\tau_{n}^{\mathrm{SRH}}$ and $\tau_{p}^{\mathrm{SRH}}\left(N_{\mathrm{c}, \mathrm{v}}\right.$ - density of states of conduction, valence band; $E_{\mathrm{t}}$ - mid gap defect energy). SRH lifetimes are different for electrons and holes but the SRH recombination rate is usually dominated by the minority carrier lifetime. We assume $\tau_{\mathrm{nr}}=\tau_{n}^{\mathrm{SRH}}=\tau_{p}^{\mathrm{SRH}}$ in the following. The nonradiative carrier lifetime $\tau_{\mathrm{nr}}$ is a crucial material parameter for GaN-based devices. The defect density and nonradiative lifetime depend on the substrate used and on the growth quality. Since SRH lifetimes are hard to predict, we assume a common value of $\tau_{\mathrm{nr}}=1 \mathrm{~ns}$ in our simulations. The SRH lifetime in quantum wells is of particular importance and it is sometimes employed as a fit parameter to find agreement with experimental characteristics [14].

Our model includes Fermi statistics and thermionic emission of carriers at hetero-interfaces [15]. The conduction band offset $\Delta E_{\mathrm{c}}$ of hetero-interfaces is one of the critical parameters in our simulation as it controls electron confinement to the active region [16]. An offset ratio $\Delta E_{\mathrm{c}} / \Delta E_{\mathrm{g}}$ of 0.7 is assumed for our device which represents an average of reported values [17-19]. 


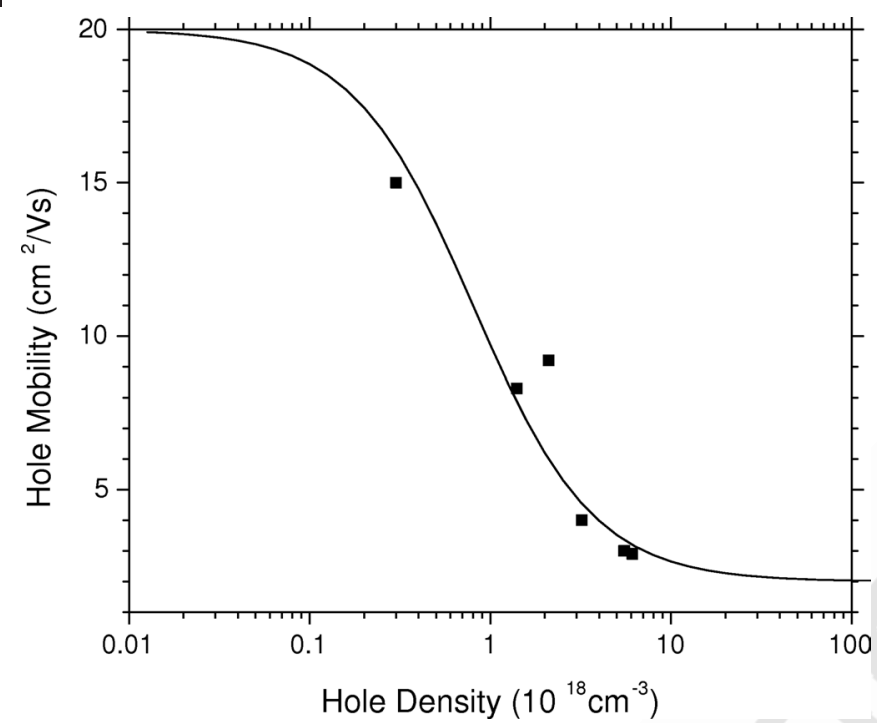

Fig. 19.3 GaN hole mobility vs hole density. The solid line gives our fit of Eq. (19.8) to measurements (dots) [22] using the parameters in Table 19.2.

The doping data in Table 19.1 give the actual densities of free carriers. While the Si donor exhibits a low ionization energy, the $\mathrm{Mg}$ acceptor has a high activation energy of about $170 \mathrm{meV}$ [20]. Large $\mathrm{Mg}$ densities near $10^{20} \mathrm{~cm}^{-3}$ are required to obtain free hole densities above $10^{18} \mathrm{~cm}^{-3}$ [21]. The high defect density contributes to a very low hole mobility, which is another obstacle to GaN device operation. The hole mobility measured on Mg-doped MOCVDgrown GaN layers is less than $20 \mathrm{~cm}^{2} \mathrm{~V}^{-1} \mathrm{~s}^{-1}$ and it decreases with higher doping density [22]. We employ the Caughey-Thomas approximation for the mobility as function of carrier density [23]

$$
\mu(N)=\mu_{\min }+\frac{\mu_{\max }-\mu_{\min }}{1+\left(N / N_{\text {ref }}\right)^{\alpha}}
$$

Tab. 19.2 GaN mobility parameters for Eq. (19.8).

\begin{tabular}{|c|c|c|c|c|}
\hline $\begin{array}{l}\text { Parameter } \\
\text { Unit }\end{array}$ & $\begin{array}{l}\mu_{\max } \\
\mathbf{c m}^{2} \mathbf{V}^{-1} \mathbf{s}^{-1}\end{array}$ & $\mu_{\min } \mathbf{c m}^{2} \mathbf{v}^{-1} \mathbf{s}^{-1}$ & $\begin{array}{l}N_{\text {ref }} \\
\mathrm{cm}^{-3}\end{array}$ & $\alpha$ \\
\hline Electrons [24] & 1405 & 80 & $0.771 \times 10^{17}$ & 0.71 \\
\hline Holes & 20 & 2 & $8 \times 10^{17}$ & 1.3 \\
\hline
\end{tabular}

which can be fitted to mobility measurements using the parameters in Table 19.2. Figure 19.3 plots Eq. (19.8) for holes in comparison to mobility measurements on Mg-doped GaN layers grown by MOCVD. We here exclude 
Tab. 19.3 Parameters for nitride wurtzite semiconductors at room temperature.

\begin{tabular}{lllrrr}
\hline Parameter & Symbol & Unit & InN & GaN & \multicolumn{1}{c}{ AIN } \\
\hline Electron eff. mass (c-axis) & $m_{\mathrm{c}}^{z}$ & $m_{0}$ & 0.11 & 0.20 & 0.33 \\
Electron eff. mass (transversal) & $m_{\mathrm{c}}^{t}$ & $m_{0}$ & 0.11 & 0.18 & 0.25 \\
Hole eff. mass parameter & $A_{1}$ & - & -9.24 & -7.24 & -3.95 \\
Hole eff. mass parameter & $A_{2}$ & - & -0.60 & -0.51 & -0.27 \\
Hole eff. mass parameter & $A_{3}$ & - & 8.68 & 6.73 & 3.68 \\
Hole eff. mass parameter & $A_{4}$ & - & -4.34 & -3.36 & -1.84 \\
Hole eff. mass parameter & $A_{5}$ & - & -4.32 & -3.35 & -1.92 \\
Hole eff. mass parameter & $A_{6}$ & - & -6.08 & -4.72 & -2.91 \\
Valence band reference level & $E_{\mathrm{V}}$ & $\mathrm{eV}$ & -1.59 & -2.64 & -3.44 \\
Direct band gap & $E_{\mathrm{g}}$ & $\mathrm{eV}$ & 0.8 & 3.42 & 6.28 \\
Spin-orbit split energy & $\Delta_{\mathrm{so}}$ & $\mathrm{eV}$ & 0.001 & 0.014 & 0.019 \\
Crystal-field split energy & $\Delta_{\mathrm{cr}}$ & $\mathrm{eV}$ & 0.041 & 0.019 & -0.164 \\
Lattice constant & $a_{0}$ & $\AA$ & 3.548 & 3.189 & 3.112 \\
Elastic constant & $C_{33}$ & $\mathrm{GPa}$ & 200 & 392 & 382 \\
Elastic constant & $C_{13}$ & $\mathrm{GPa}$ & 94 & 100 & 127 \\
Deform. potential $\left(E_{\mathrm{c}}\right)$ & $a_{\mathrm{c}}$ & $\mathrm{eV}$ & & -4.08 & \\
Deform. potential & $D_{1}$ & $\mathrm{eV}$ & & -0.89 & \\
Deform. potential & $D_{2}$ & $\mathrm{eV}$ & & 4.27 & \\
Deform. potential & $D_{3}$ & $\mathrm{eV}$ & & 5.18 & \\
Deform. potential & $D_{4}$ & $\mathrm{eV}$ & & -2.59 & \\
Dielectric constant & $\varepsilon$ & - & 15.0 & 9.5 & 8.5 \\
\hline
\end{tabular}

Note. $\Delta_{\mathrm{cr}}=\Delta_{1}, \Delta_{\mathrm{so}}=3 \Delta_{2}=3 \Delta_{3}, a_{\mathrm{c}}=a / 2$

measurements of C-doped or MBE-grown GaN layers [25] which lead to inappropriate fit parameters as in [26]. In ternary and MQW layers, the mobilities are further reduced by additional scattering mechanisms, that are related to alloy disorder, interface roughness, or compositional fluctuations. These effects are hard to predict and we assume an electron mobility of $100 \mathrm{~cm}^{2} \mathrm{~V}^{-1} \mathrm{~s}^{-1}$ and a hole mobility of $5 \mathrm{~cm}^{2} \mathrm{~V}^{-1} \mathrm{~s}^{-1}$ for all ternary layers. In addition to the low hole conductivity, a high contact resistance may significantly increase the device bias of GaN-based light emitters.

19.3.2

Electron Band Structure

PICS3D includes the $6 \times 6 \vec{k} \cdot \overrightarrow{\mathbf{p}}$ model for the valence-band structure of wurtzite semiconductors as developed by Chuang and Chang $[27,28]$, which is summarized in this section with an emphasis on strain effects. The three valence bands are referred to as heavy-hole (hh), light-hole (lh), and crystal-field split-hole (ch) band. Material parameters are listed in Table 19.3. 


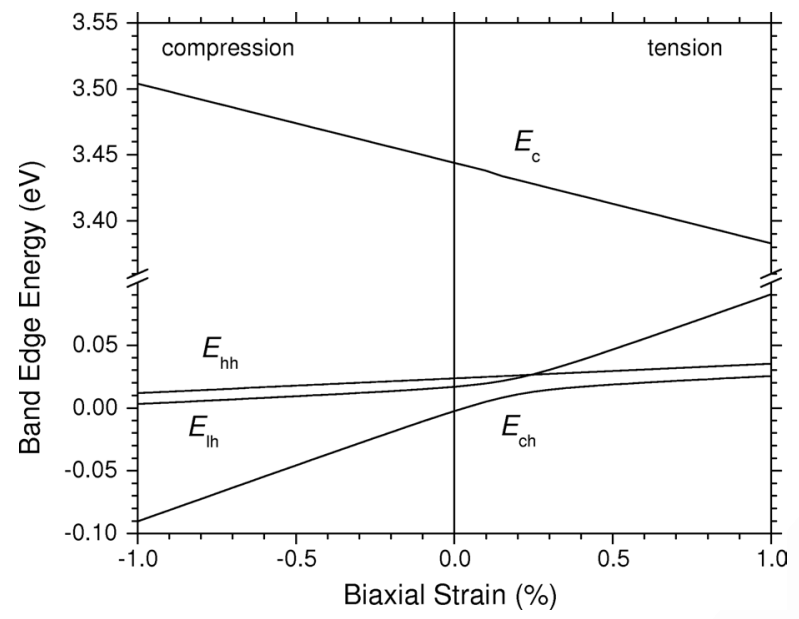

Fig. 19.4 GaN band-edge shift with strain.

The epitaxial growth of nitride devices is typically along the $c$-axis of the wurtzite crystal, which is parallel to the $z$-axis in our coordinate system. The natural $\operatorname{In}_{x} \mathrm{Ga}_{1-x} \mathrm{~N}$ lattice constant $a_{0}(x)$ is reduced to that of the GaN substrate, $a_{\mathrm{s}}$, imposing biaxial compressive strain in the transverse plane

$$
e_{\mathrm{t}}=\frac{a_{\mathrm{s}}-a_{0}}{a_{0}}
$$

and tensile strain in the growth direction

$$
e_{z}=-2 \frac{C_{13}}{C_{33}} e_{t}
$$

The nondiagonal elements of the strain tensor are zero. The valence band edge energies are

$$
\begin{aligned}
& E_{\mathrm{hh}}=E_{\mathrm{v}}+\Delta_{1}+\Delta_{2}+\theta_{e}+\lambda_{e} \\
& E_{\mathrm{lh}}=E_{\mathrm{v}}+\frac{\Delta_{1}-\Delta_{2}+\theta_{e}}{2}+\lambda_{e}+\sqrt{\left(\frac{\Delta_{1}-\Delta_{2}+\theta_{e}}{2}\right)^{2}+2 \Delta_{3}^{2}} \\
& E_{\mathrm{ch}}=E_{\mathrm{v}}+\frac{\Delta_{1}-\Delta_{2}+\theta_{e}}{2}+\lambda_{e}-\sqrt{\left(\frac{\Delta_{1}-\Delta_{2}+\theta_{e}}{2}\right)^{2}+2 \Delta_{3}^{2}}
\end{aligned}
$$

with the average valence band edge $E_{\mathrm{v}}$ and

$$
\begin{aligned}
& \theta_{e}=D_{3} e_{z}+2 D_{4} e_{t} \\
& \lambda_{e}=D_{1} e_{z}+2 D_{2} e_{t}
\end{aligned}
$$

Without strain, the spin-orbit interaction leads to only slight separations between the three valence band edges. Figure 19.4 shows the influence of strain. 
In contrast to traditional zinc blende III-V compounds like GaAs, the GaN light hole and heavy hole bands hardly separate under compressive strain. This contributes to the high quantum well carrier densities and threshold current densities observed with GaN-based lasers [22], which are much higher than with GaAs-based lasers.

The conduction band edge is calculated from

$$
E_{\mathrm{c}}=E_{\mathrm{v}}+E_{\mathrm{g}}+P_{\mathrm{ce}}
$$

with the energy band gap $E_{\mathrm{g}}$ and the hydrostatic energy shift

$$
P_{\mathrm{ce}}=a_{\mathrm{cz} z} e_{z}+2 a_{\mathrm{c} t} e_{t}
$$

The hydrostatic deformation potential is anisotropic $\left(a_{z}, a_{t}\right)$ and half of the deformation is assumed to affect the conduction band $\left(a_{\mathrm{cz}}, a_{\mathrm{ct}}\right)$. The band gaps of $\operatorname{In}_{x} \mathrm{Ga}_{1-x} \mathrm{~N}$ and $\mathrm{Al}_{x} \mathrm{Ga}_{1-x} \mathrm{~N}$ are known to deviate from the linear Vegard law, and they are approximated by

$$
E_{\mathrm{g}}^{0}(x)=x E_{\mathrm{g}}^{0}(\mathrm{AlN} \text { or InN })+(1-x) E_{\mathrm{g}}^{0}(\mathrm{GaN})-x(1-x) C_{g}
$$

using the bowing parameter $C_{g}$. A wide range of bowing parameters has been reported [12]. For unstrained layers with a low mole fraction of the alloy element we adopt $C_{g}=2.6 \mathrm{eV}$ for InGaN [29] and $C_{g}=1.3 \mathrm{eV}$ for AlGaN [30].

The dispersion $E_{\mathrm{c}}(\vec{k})$ of the conduction band can be characterized by a parabolic band model with electron effective masses $m_{\mathrm{c}}^{t}$ and $m_{\mathrm{c}}^{z}$ perpendicular and parallel to the $c$-growth direction, respectively. The three valence bands are nonparabolic. Near the $\Gamma$ point, the hole effective masses can be approximated as

$$
\begin{aligned}
m_{\mathrm{hh}}^{z} & =-m_{0}\left(A_{1}+A_{3}\right)^{-1} \\
m_{\mathrm{hh}}^{t} & =-m_{0}\left(A_{2}+A_{4}\right)^{-1} \\
m_{\mathrm{lh}}^{z} & =-m_{0}\left[A_{1}+\left(\frac{E_{\mathrm{lh}}-\lambda_{e}}{E_{\mathrm{lh}}-E_{\mathrm{ch}}}\right) A_{3}\right]^{-1} \\
m_{\mathrm{lh}}^{t} & =-m_{0}\left[A_{2}+\left(\frac{E_{\mathrm{lh}}-\lambda_{e}}{E_{\mathrm{lh}}-E_{\mathrm{ch}}}\right) A_{4}\right]^{-1} \\
m_{\mathrm{ch}}^{z} & =-m_{0}\left[A_{1}+\left(\frac{E_{\mathrm{ch}}-\lambda_{e}}{E_{\mathrm{ch}}-E_{\mathrm{lh}}}\right) A_{3}\right]^{-1} \\
m_{\mathrm{ch}}^{t} & =-m_{0}\left[A_{2}+\left(\frac{E_{\mathrm{ch}}-\lambda_{e}}{E_{\mathrm{ch}}-E_{\mathrm{lh}}}\right) A_{4}\right]^{-1}
\end{aligned}
$$

using the hole effective mass parameters $A_{i}$ given in Table $19.3\left(m_{0}-\right.$ free electron mass). More details on the calculation procedure for quantum well valence bands are given in [28]. Figure 19.5 shows the electron band structure calculated for our $\operatorname{In}_{0.1} \mathrm{Ga}_{0.9} \mathrm{~N}$ quantum well. 


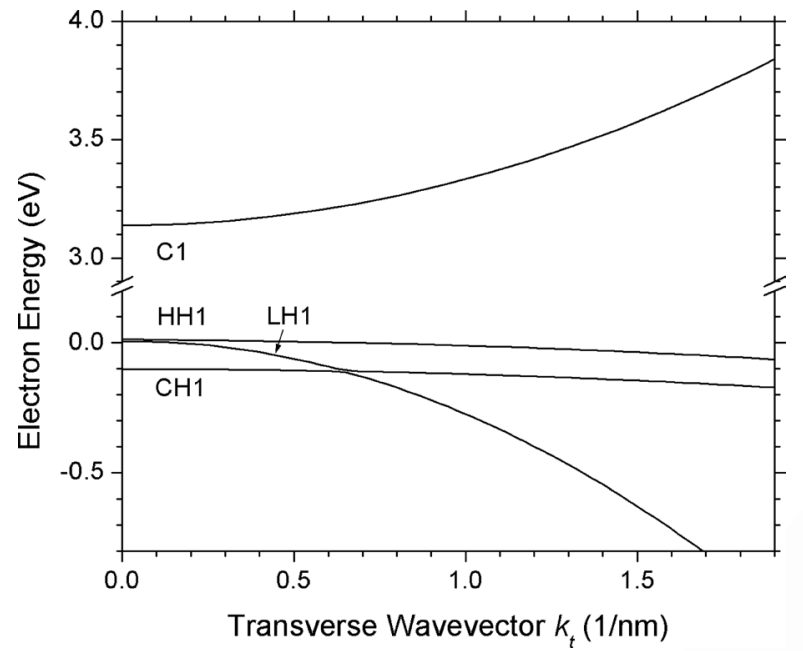

Fig. 19.5 Quantum well band structure within the transverse plane (lowest levels).

19.3.3

\section{Built-in Polarization}

Spontaneous and piezoelectric polarization of nitride compounds is larger than in other III-V semiconductors. It depends on the compound's composition. Net polarization charges remain at each hetero-interface. We here use the nonlinear model described in [31]. Accordingly, the spontaneous polarization $P_{\mathrm{sp}}\left[\mathrm{C} \mathrm{m}^{-2}\right]$ is calculated as

$$
\begin{aligned}
& P_{\mathrm{sp}}\left(\mathrm{Al}_{\mathrm{x}} \mathrm{Ga}_{1-\mathrm{x}} \mathrm{N}\right)=-0.090 \mathrm{x}-0.034(1-\mathrm{x})+0.019 \mathrm{x}(1-\mathrm{x}) \\
& P_{\mathrm{sp}}\left(\mathrm{In}_{\mathrm{x}} \mathrm{Ga}_{1-\mathrm{x}} \mathrm{N}\right)=-0.042 \mathrm{x}-0.034(1-\mathrm{x})+0.038 \mathrm{x}(1-\mathrm{x})
\end{aligned}
$$

For binary compounds, the piezoelectric polarization $P_{\mathrm{pz}}\left[\mathrm{C} \mathrm{m}^{-2}\right]$ is given as nonlinear functions of the transverse strain $e_{t}$ by

$$
\begin{aligned}
& P_{\mathrm{pz}}(\mathrm{GaN})=-0.918 \mathrm{e}_{\mathrm{t}}+9.541 \mathrm{e}_{\mathrm{t}}^{2} \\
& P_{\mathrm{pz}}(\mathrm{InN})=-1.373 \mathrm{e}_{\mathrm{t}}+7.559 \mathrm{e}_{\mathrm{t}}^{2} \\
& P_{\mathrm{pz}}(\mathrm{AlN})=-1.808 \mathrm{e}_{\mathrm{t}}-7.888 \mathrm{e}_{\mathrm{t}}^{2}\left(\mathrm{e}_{\mathrm{t}}>0\right) \\
& P_{\mathrm{pz}}(\mathrm{AlN})=-1.808 \mathrm{e}_{\mathrm{t}}+5.624 \mathrm{e}_{\mathrm{t}}^{2}\left(\mathrm{e}_{\mathrm{t}}<0\right)
\end{aligned}
$$

and it is linearly interpolated for ternary compounds. Spontaneous and piezoelectric polarization add up and result in a strong built-in field (Fig. 19.6). In PICS3D, the built-in polarization is represented by a fixed surface charge density. At hetero-interfaces, the difference of these surface charge densities gives 
Tab. 19.4 Built-in polarization charges at VCSEL interfaces.

\begin{tabular}{lr}
\hline Interface & Net density \\
\hline $\mathrm{GaN} / \mathrm{In}_{0.035} \mathrm{Ga}_{0.965} \mathrm{~N}$ & $-3.2 \times 10^{12} \mathrm{~cm}^{-2}$ \\
$\mathrm{In}_{0.035} \mathrm{Ga}_{0.965} \mathrm{~N} / \mathrm{In}_{0.1} \mathrm{Ga}_{0.9} \mathrm{~N}$ & $-6.5 \times 10^{12} \mathrm{~cm}^{-2}$ \\
$\mathrm{In}_{0.1} \mathrm{Ga}_{0.9} \mathrm{~N} / \mathrm{In}_{0.035} \mathrm{Ga}_{0.965} \mathrm{~N}$ & $+6.5 \times 10^{12} \mathrm{~cm}^{-2}$ \\
$\mathrm{In}_{0.035} \mathrm{Ga}_{0.965} \mathrm{~N} / \mathrm{Al}_{0.18} \mathrm{Ga}_{0.82} \mathrm{~N}$ & $+11.0 \times 10^{12} \mathrm{~cm}^{-2}$ \\
$\mathrm{Al}_{0.18} \mathrm{Ga}_{0.82} \mathrm{~N} / \mathrm{GaN}$ & $-7.8 \times 10^{12} \mathrm{~cm}^{-2}$ \\
\hline
\end{tabular}

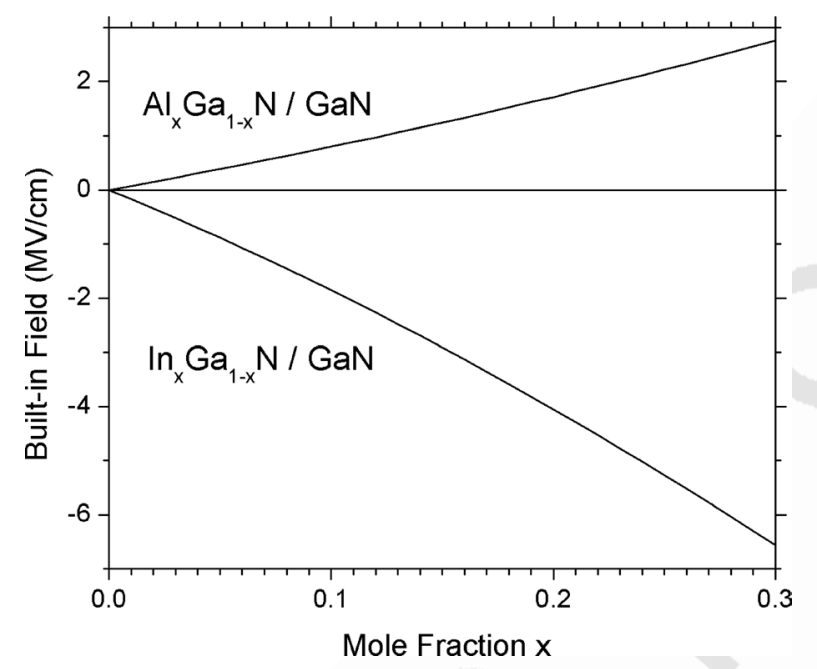

Fig. 19.6 Net built-in field in thin ternary layers grown on GaN.

the net polarization charge density which is listed in Table 19.4 for the interfaces in our device.

Built-in polarization fields are expected to strongly affect the VCSEL performance [32]. Within the quantum wells, the polarization field separates electrons and holes, thereby reducing stimulated and spontaneous emission. However, experimental investigations of InGaN quantum wells often result in weaker built-in fields than predicted, ranging from $20 \%$ [33] to $80 \%$ [34] of the theoretical value, with typical results near 50\% [35]. This broad variation has been attributed to partial compensation of the polarization field by fixed defect and interface charges [36] or to inappropriate analysis of measured data [37]. On the other hand, the theoretical polarization formulas may deviate from reality, especially for InGaN, as only AlGaN measurements have been used for validation [31]. Since the actual magnitude of the built-in polarization in our device is unknown, we are using the polarization charges as a variable in some of our simulations. 
19.3.4

\section{Photon Generation in the Quantum Wells}

The carrier recombination mechanisms in InGaN quantum wells are not yet fully understood. The small impact of the high defect density still puzzles many researchers. Indium segregation, defect self-screening, or a short hole diffusion length may play an important role. Essential quantum well parameters like nonradiative carrier lifetime and net polarization field are not exactly known. All these uncertainties make it difficult to verify models for the photon generation rates. We here use the same free-carrier model which previously resulted in good agreement with measurements on InGaN/GaN inplane lasers [11]. More sophisticated many-body models are presented in [38].

The net optical gain $g(h v)$ of stimulated carrier recombination (photon emission) and carrier generation (photon absorption) is a function of the photon energy $h v$. For transitions between parabolic subbands $i$ and $j$ it is given by

$$
g(h v)=\left(\frac{q^{2} h}{2 m_{0}^{2} \varepsilon_{0} n_{\mathrm{r}} c}\right)\left(\frac{1}{h v}\right) \sum_{i, j} \int|M|^{2} D_{\mathrm{r}}\left(f_{\mathrm{c}}-f_{\mathrm{v}}\right) L d E
$$

with Planck's constant $h$, the refractive index $n_{r}$, the photon velocity $c$, and the transition energy $E$. The Fermi functions $f_{\mathrm{C}}$ and $f_{\mathrm{V}}$ for the electron population in the conduction and valence subband, respectively, determine whether more photons are generated $\left(f_{\mathrm{c}}>f_{\mathrm{V}}\right)$ or absorbed $\left(f_{\mathrm{c}}<f_{\mathrm{V}}\right)$. $D_{\mathrm{r}}$ is the reduced density of states between the two subbands. Carrier scattering within each band causes an energy broadening which is considered by the Lorentzian lineshape function

$$
L(h v-E)=\frac{1}{\pi} \frac{\Gamma_{\mathrm{s}}}{(h v-E)^{2}+\Gamma_{\mathrm{s}}^{2}}
$$

with the half-width $\Gamma_{\mathrm{s}}=6.6 \mathrm{meV}$ (scattering time $\tau_{\mathrm{s}}=0.1 \mathrm{ps}$ ) [39]. $|M|^{2}$ is the momentum matrix element and it gives the transition strength. Its computation is based on the $\vec{k} \cdot \overrightarrow{\mathbf{p}}$ electron band structure model outlined above. The matrix element depends on the photon polarization which has two principal directions: parallel (TE) and perpendicular (TM) to the quantum well plane. For a typical VCSEL, only the TE polarization contributes to lasing. However, the TM polarization needs to be included in the computation of spontaneous photon emission. For a quantum well grown in the hexagonal $c$ direction, the energy dependent matrix elements for transitions involving heavy (hh), 
light (lh), and crystal-field holes (ch), respectively, can be written as

$$
\begin{aligned}
\left|M_{\mathrm{hh}}^{\mathrm{TE}}\right|^{2} & =\frac{3}{2} O_{i j}\left(M_{\mathrm{b}}^{\mathrm{TE}}\right)^{2} \\
\left|M_{\mathrm{lh}}^{\mathrm{TE}}\right|^{2} & =\frac{3}{2} \cos ^{2}\left(\theta_{\mathrm{k}}\right) O_{i j}\left(M_{\mathrm{b}}^{\mathrm{TE}}\right)^{2} \\
\left|M_{\mathrm{ch}}^{\mathrm{TE}}\right|^{2} & =0 \\
\left|M_{\mathrm{hh}}^{\mathrm{TM}}\right|^{2} & =0 \\
\left|M_{\mathrm{lh}}^{\mathrm{TM}}\right|^{2} & =3 \sin ^{2}\left(\theta_{\mathrm{k}}\right) O_{i j}\left(M_{\mathrm{b}}^{\mathrm{TM}}\right)^{2} \\
\left|M_{\mathrm{ch}}^{\mathrm{TM}}\right|^{2} & =3 O_{i j}\left(M_{\mathrm{b}}^{\mathrm{TM}}\right)^{2}
\end{aligned}
$$

The overlap integral $O_{i j}$ of the electron and hole wavefunctions can assume values between 0 and 1 . At the $\Gamma$ point, $O_{i j}$ is nonzero only for subbands with the same quantum number. Away from the $\Gamma$ point, $O_{i j}$ may be nonzero for any transition. The angle $\theta_{\mathrm{k}}$ of the electron wave vector $\vec{k}$ to the $k_{z}$ direction introduces an additional energy dependence to the matrix element, with $\cos \left(\theta_{\mathrm{k}}\right)=1$ at the $\Gamma$ point. The bulk momentum matrix elements are given by [39]

$$
\begin{aligned}
& \left(M_{\mathrm{b}}^{\mathrm{TE}}\right)^{2}=\frac{m_{0}}{6}\left(\frac{m_{0}}{m_{\mathrm{c}}^{t}}-1\right) \frac{E_{\mathrm{g}}\left[\left(E_{\mathrm{g}}+\Delta_{1}+\Delta_{2}\right)\left(E_{\mathrm{g}}+2 \Delta_{2}\right)-2 \Delta_{3}^{2}\right]}{\left(E_{\mathrm{g}}+\Delta_{1}+\Delta_{2}\right)\left(E_{\mathrm{g}}+\Delta_{2}\right)-\Delta_{3}^{2}} \\
& \left(M_{\mathrm{b}}^{\mathrm{TM}}\right)^{2}=\frac{m_{0}}{6}\left(\frac{m_{0}}{m_{\mathrm{c}}^{z}}-1\right) \frac{\left(E_{\mathrm{g}}+\Delta_{1}+\Delta_{2}\right)\left(E_{\mathrm{g}}+2 \Delta_{2}\right)-2 \Delta_{3}^{2}}{E_{\mathrm{g}}+2 \Delta_{2}}
\end{aligned}
$$

Note that the bulk electron mass is different in the transversal $\left(m_{\mathrm{c}}^{t}\right)$ and parallel directions $\left(m_{\mathrm{c}}^{z}\right)$ relative to the hexagonal $c$-axis. The material parameters are given in Table 19.3.

Figure 19.7 plots the calculated gain spectra for our quantum well at different carrier densities. Built-in polarization is not considered here. However, relatively large densities are required to obtain positive gain. A second gain peak from transitions between higher quantum levels emerges with carrier densities above $5 \times 10^{19} \mathrm{~cm}^{-3}$. The gain peaks' red-shift is caused by band gap renormalization which is included as a function of the quantum well $2 \mathrm{D}$ carrier density $N_{2 D}$ using the simple formula

$$
\Delta E_{\mathrm{g}}=-\zeta N_{2 \mathrm{D}}^{1 / 3}
$$

with $\zeta=6 \times 10^{-6} \mathrm{eV} \mathrm{cm}^{2 / 3}$ [40].

The spontaneous photon emission rate is calculated as

$$
r_{\mathrm{sp}}(h v)=\left(\frac{q^{2} h}{2 m_{0}^{2} \varepsilon \varepsilon_{0}}\right)\left(\frac{1}{h v}\right) \sum_{i, j} \int|\bar{M}|^{2} D_{\mathrm{opt}} D_{\mathrm{r}} f_{\mathrm{c}}\left(1-f_{\mathrm{v}}\right) L d E
$$




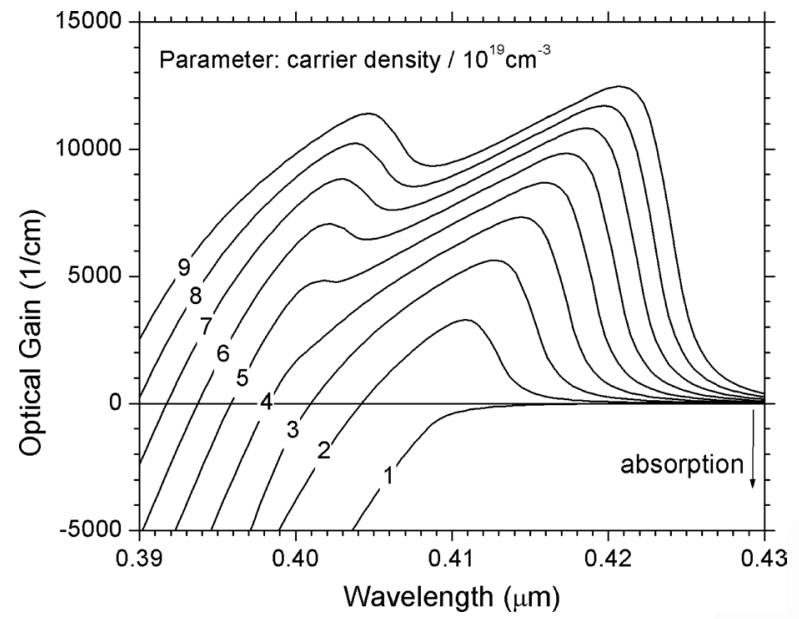

Fig. 19.7 Gain spectra at different carrier densities.

with the density of photon states $D_{\text {opt }}$ and the polarization-averaged matrix element $|\bar{M}|^{2}$. The Fermi factor $f_{\mathrm{c}}\left(1-f_{\mathrm{v}}\right)$ is different from that in the gain formula (19.31), i.e., the spontaneous emission spectrum peaks at slightly higher photon energies than the gain spectrum.

19.3.5

\section{Optical Mode}

Calculation of the internal optical field is one of the most challenging tasks of VCSEL simulations. A precise optical analysis requires sophisticated solutions to Maxwell's equations for an open resonator. Various numerical models have been developed, most of which are reviewed in [41]. As this chapter focuses on electronic effects, we use a simplified optical model and restrict our simulation to the fundamental lasing mode. Higher-order VCSEL modes are investigated in the next chapter.

Our model is based on the effective index method [42] and it decouples the optical fields in the vertical $(z)$ and the transverse $(r)$ direction. The fundamental mode profile is approximated by a zeroth-order Bessel function which is a solution to the reduced scalar Helmholtz equation for the lateral optical field $\Phi(r)$ in cylindrical waveguides

$$
\frac{d^{2} \Phi}{d r^{2}}+\frac{1}{r} \frac{d \Phi}{d r}+\left(k^{2}-k_{z}^{2}\right) \Phi=0
$$

with the optical wave number

$$
k=n_{\mathrm{r}} \frac{2 \pi}{\lambda_{0}}
$$




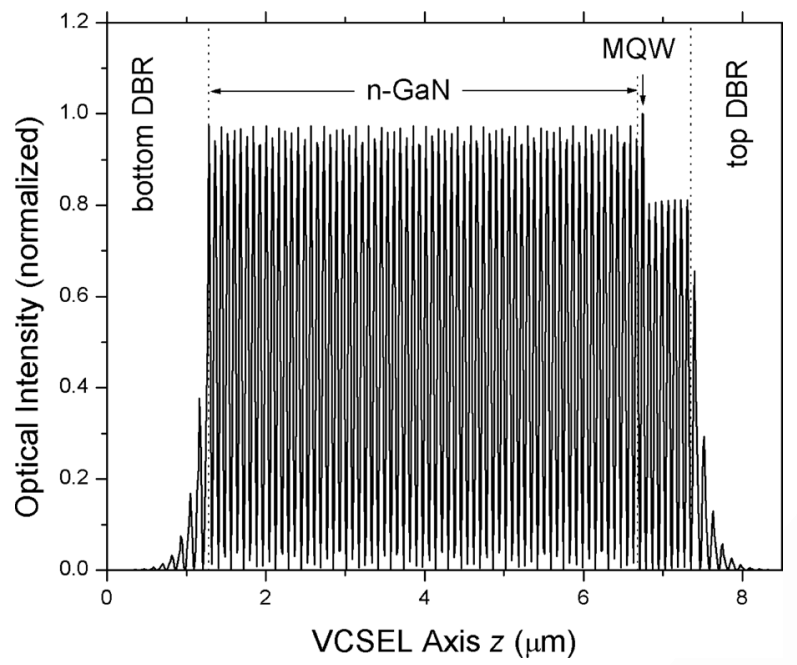

Fig. 19.8 Vertical standing wave.

( $n_{\mathrm{r}}$ - refractive index, $\lambda_{0}$ - free space wavelength). The mode diameter is given by the ring contact aperture of $12 \mu \mathrm{m}$ (see Fig. 19.10 below). The refractive index is calculated using the model in [43] which shows good agreement with measurement. The resulting index data at $400 \mathrm{~nm}$ wavelength are listed in Table 19.1. A modal optical loss of $\alpha_{\mathrm{i}}=20 \mathrm{~cm}^{-1}$ is assumed.

In the vertical direction, the transmission matrix method [9] is utilized to obtain the standing optical wave for our large VCSEL cavity (Fig. 19.8). Due to the quarter-wavelength thickness of the DBR layers, null or peak of the optical field are located at each DBR interface. For maximum modal gain, the MQW region is placed at a peak of the standing wave. Due to the thick n-GaN layer, many vertical modes are allowed in our VCSEL with a narrow mode spacing of a few nanometers (Fig. 19.9). This is unusual for VCSELs, but it eliminates a crucial VCSEL design issue, namely the alignment of mode wavelength and optical gain spectrum [8] (cf. Fig. 19.7). In our VCSEL, the optical mode nearest to the gain peak is expected to lase so that the shape of the gain spectrum is less important. The peak gain can reasonably be approximated by a freecarrier model as described above.

\section{4}

\section{Simulation Results and Device Analysis}

In the following, we utilize our VCSEL simulation to analyze various internal device processes, including current confinement, polarization effects, and electron leakage. Some of those processes limit the device performance and 


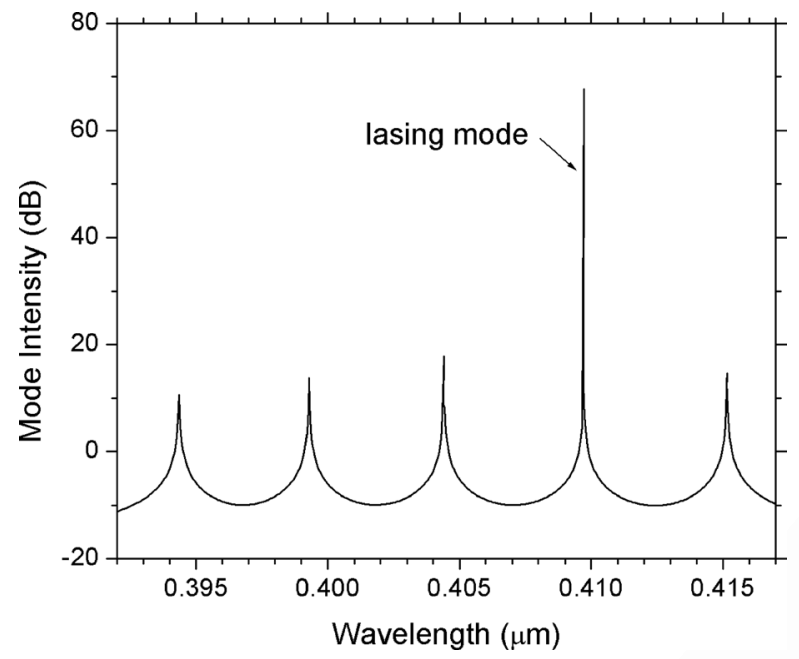

Fig. 19.9 Mode spectrum.

contribute to the fact that no lasing was observed in the experimental investigation of our example device [6].

19.4.1

\section{Current Confinement}

The optical mode size in our device is laterally confined by the top ring contact with about $12 \mu \mathrm{m}$ aperture (cf. Fig. 19.2). Hole injection is provided by the top ITO layer with manufactured apertures between $2 \mu \mathrm{m}$ and $10 \mu \mathrm{m}$. Due to the poor hole mobility, the lateral hole spreading is very small and the carrier profile within the active layers is well confined to the injection aperture. Figure 19.10 illustrates this situation for the device with $2 \mu \mathrm{m}$ ITO aperture. Due to the narrowly confined carrier density, positive optical gain is only provided for $r<1.5 \mu \mathrm{m}$. Beyond that radius, the optical mode experiences strong quantum well absorption leading to a net modal gain below zero. Thus, the $2 \mu \mathrm{m}$ VCSEL never reaches the lasing threshold in our simulation. In the following, we therefore focus our investigation on VCSELs with $10 \mu \mathrm{m}$ injection aperture, which seem to have a better chance to lase.

\subsection{2}

\section{Polarization Effects}

The built-in polarization strongly deforms the energy band diagram of our MQW active region. Figure 19.11 compares the MQW band diagrams as calculated at forward bias with and without the built-in polarization charges given in Table 19.4. Without polarization, the quantum wells are almost rectangu- 


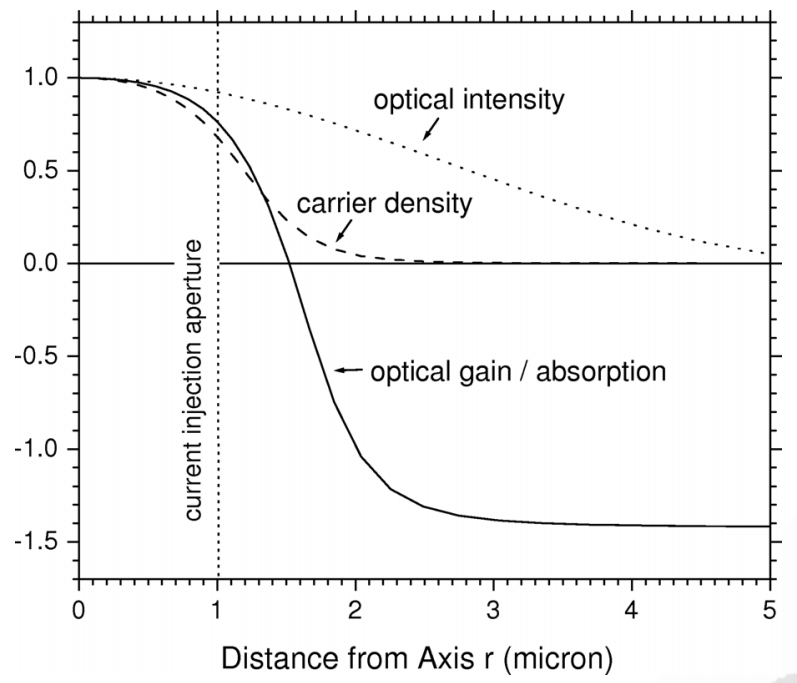

Fig. 19.10 Normalized lateral profiles within the quantum well for $2 \mu \mathrm{m}$ current injection aperture.

lar and the AlGaN layer imposes a considerable energy barrier of $250 \mathrm{meV}$ on electrons trying to leak out of the MQW active region. With full polarization, the energy band diagram is significantly deformed. This deformation is even more remarkable considering the high current density of $j=50 \mathrm{kA} \mathrm{cm}^{-2}$ used in this calculation, which is 16 times higher than the threshold current density of similar edge-emitting lasers [11]. Surprisingly, even with strong carrier injection, the built-in polarization field is not completely screened as often assumed for laser operation.

The corresponding electrostatic field profile is plotted in Fig. 19.12. The polarization charge densities at the MQW interfaces translate into a built-in quantum well field of $1.8 \mathrm{MV} \mathrm{cm}^{-1}$. The actual electrostatic field within the quantum wells is about $0.5 \mathrm{MV} \mathrm{cm}^{-1}$ due to partial screening by electrons and holes. Figure 19.13 gives the carrier density profile. The built-in polarization clearly leads to a separation of electrons and holes within the quantum wells. But even with a current density of $50 \mathrm{kA} \mathrm{cm}^{-2}$, the injected quantum well carrier density is not large enough to completely screen the built-in field. This can be easily understood by converting the interface charge densities given in Table 19.4 into a uniform quantum well carrier density of $2.4 \times 10^{19} \mathrm{~cm}^{-3}$ needed for full screening. 


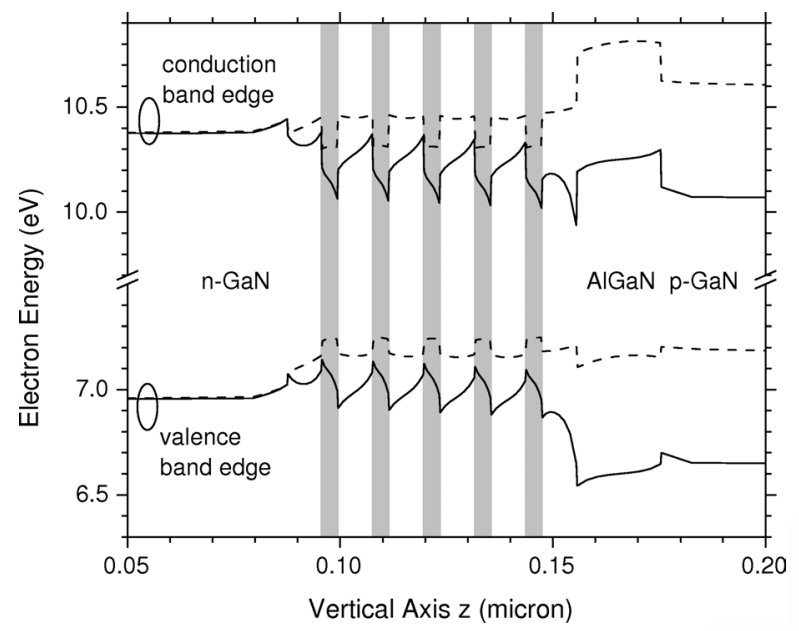

Fig. 19.11 Forward-bias MQW energy band diagram at the VCSEL axis with (solid) and without (dashed) the built-in polarization charges given in Table 19.4 (grey: quantum wells, $j=50 \mathrm{kA} \mathrm{cm}^{-2}, p_{\text {AlGaN }}=$ $\left.10^{16} \mathrm{~cm}^{-3}\right)$.

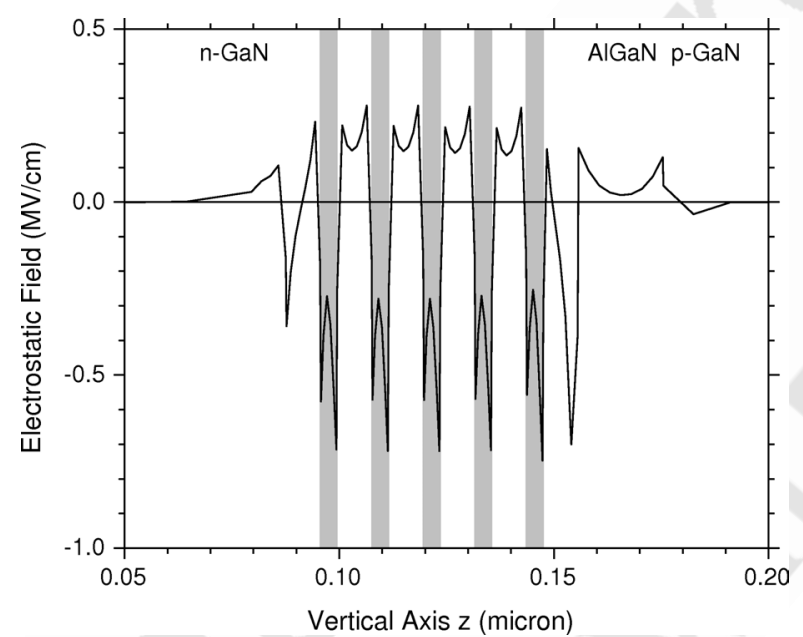

Fig. 19.12 Electrostatic field at the VCSEL axis with full polarization (grey: quantum wells, $j=50 \mathrm{kA} \mathrm{cm}^{-2}, p_{\mathrm{AlGaN}}=10^{16} \mathrm{~cm}^{-3}$ ).

\subsection{3}

\section{Threshold Current}

The separation of electrons and holes within the quantum well reduces the radiative recombination rate and leads to an increased threshold current of the laser. Figure 19.14 shows the calculated VCSEL threshold current as a function of polarization strength with $100 \%$ corresponding to the theoretically 


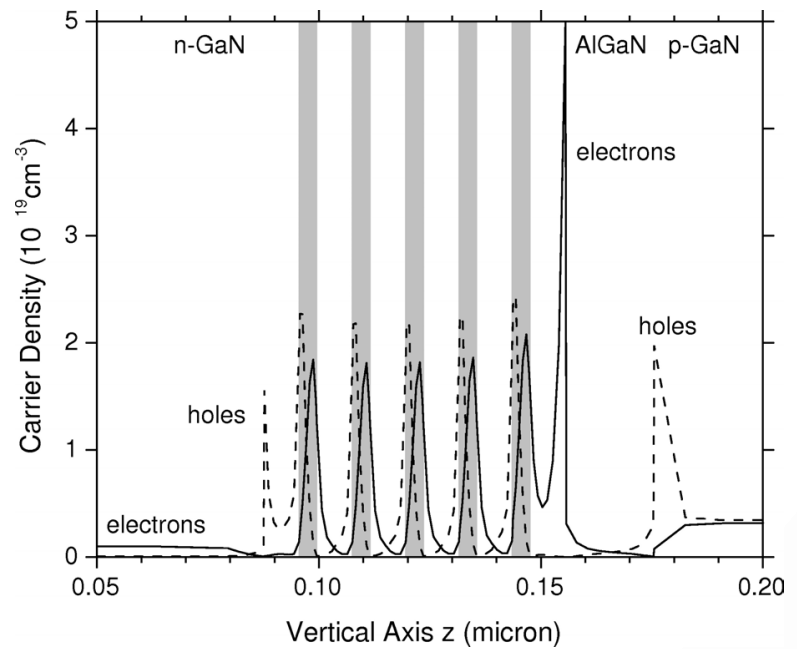

Fig. 19.13 Carrier densities at the VCSEL axis with full polarization (solid: electrons, dashed: holes, grey: quantum wells, $j=50 \mathrm{kA} \mathrm{cm}^{-2}$, $p_{\text {AlGaN }}=10^{16} \mathrm{~cm}^{-3}$ ).

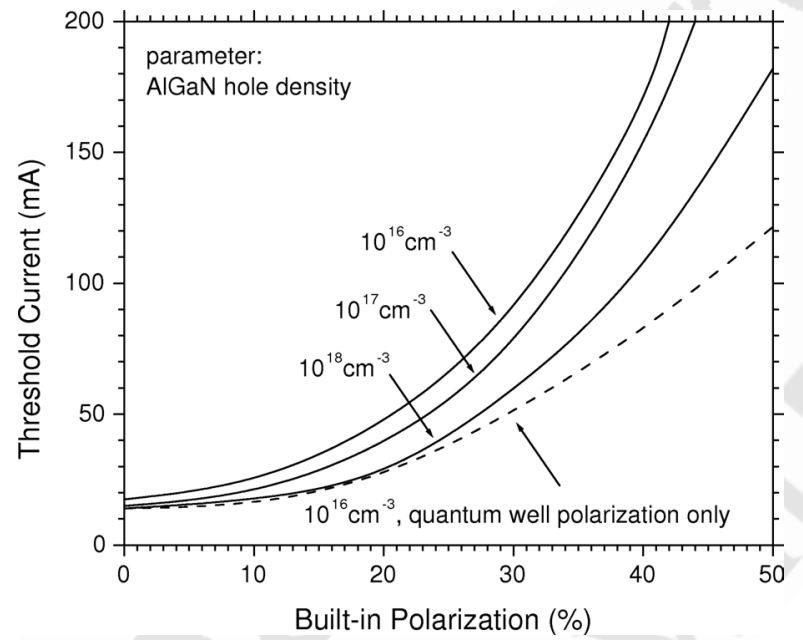

Fig. 19.14 VCSEL threshold current as function of built-in polarization strength $(100 \%=$ Table 19.4$)$.

predicted values of Table 19.4. Without polarization, the threshold current is $17 \mathrm{~mA}$. Experimentally, these VCSELs suffered from catastrophic shortcircuiting at about half that current, probably due to threading dislocations [6].

Considering only the polarization charges at the quantum well interfaces, $50 \%$ polarization enlarges the threshold current by a factor of 7 (dashed line in Fig. 19.14). This is mainly due to the reduced overlap of electron and hole wavefunctions. However, with polarization charges at all hetero-interfaces, 


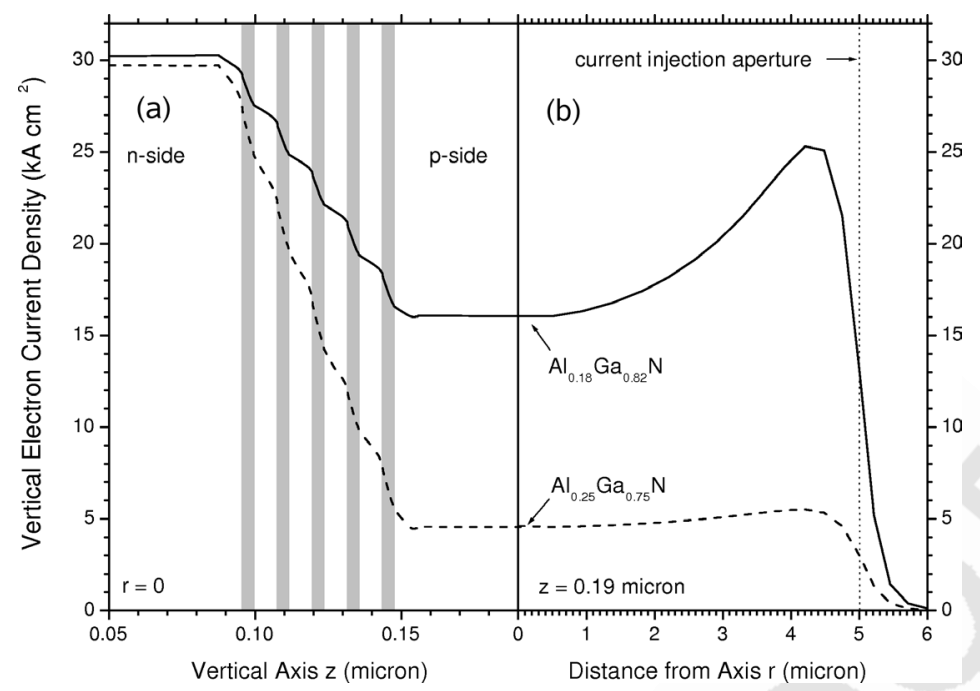

Fig. 19.15 Vertical electron current density $j_{n, z}$ with the default $\mathrm{Al}_{0.18} \mathrm{Ga}_{0.82} \mathrm{~N}$ stopper layer (solid) and with an $\mathrm{Al}_{0.25} \mathrm{Ga}_{0.75} \mathrm{~N}$ stopper layer (dashed). (a) Vertical profile $j_{n, z}(z)$ at the VCSEL axis; (b) Radial profile $j_{n, z}(r)$ above the stopper layer; (grey: quantum wells, $\left.p_{\text {AlGaN }}=10^{18} \mathrm{~cm}^{-3}\right)$.

the threshold current rises even more strongly (solid lines in Fig. 19.14). The only difference in both cases is the polarization introduced by the AlGaN stopper layer. As shown in Figs 19.11 and 19.13, the polarization charges at the In$\mathrm{GaN} / \mathrm{AlGaN}$ interface attract a large density of electrons which lead to significant band bending. Consequently, the AlGaN energy barrier is dramatically reduced allowing for strong electron leakage from the MQW. Such leakage effects are also indicated by the measured photoluminescence spectrum of this VCSEL [6] as well as by investigations on similar devices [16].

\subsection{4}

\section{AIGaN Doping}

Electron leakage is a common problem with laser diodes and stronger pdoping of the stopper layer is a typical countermeasure [44]. Figure 19.14 demonstrates the effect of AlGaN doping in our case. The free hole density is increased from $10^{16} \mathrm{~cm}^{-3}$, as used in the previous section, to $10^{18} \mathrm{~cm}^{-3}$, which is close to the maximum value achieved to date. With higher p-doping, the Al$\mathrm{GaN}$ band bending increases and so does the effective electron energy barrier imposed by the stopper layer. As our simulation results show in Fig. 19.14, raising the hole density from $10^{16} \mathrm{~cm}^{-3}$ to $10^{18} \mathrm{~cm}^{-3}$ results in a significant threshold current reduction. 


\section{AIGaN Composition}

The electron leakage can also be reduced by increasing the $\mathrm{Al}$ mole fraction of the AlGaN stopper layer. This will enlarge the AlGaN band gap but it will also lead to a higher density of polarization charges at the AlGaN interfaces. The combined effect of both changes on the electron current is illustrated in Fig. 19.15. The vertical current profile across the MQW region is shown on the left-hand side. Electrons enter the MQW from the n-side and partially recombine with holes within the quantum wells. Electrons leaving the MQW and entering the p-side constitute the leakage current. With $25 \% \mathrm{Al}$ mole fraction (dashed), the leakage current is significantly smaller than with the original $\mathrm{Al}_{0.18} \mathrm{Ga}_{0.82} \mathrm{~N}$ stopper layer (solid).

The right-hand side of Fig. 19.15 plots the lateral profile of the leakage current above the stopper layer. Its shape is similar to that of the hole current. The current peaks near the injection aperture given by the ITO contact diameter of $10 \mu \mathrm{m}$. Such current crowding reduces the quantum well carrier density in the center of the device and the modal gain, leading to an increased threshold current. Obviously, the higher $\mathrm{Al}_{0.25} \mathrm{Ga}_{0.75} \mathrm{~N}$ barrier causes a more uniform current distribution (dashed line) compared to the original stopper layer (solid line).

\section{5}

\section{Summary}

We have demonstrated how advanced simulation of GaN VCSELs can be used to gain deeper insight into internal device physics that is not available through measurements. Our simulation results help to understand performance limiting mechanisms and to improve the design of future devices.

\section{References}

1 T. E. Sale. Vertical Cavity Surface Emitting Lasers. Wiley, New York, 1995.

2 C. Wilmsen, H. Temkin, and L. A. Coldren, editors. Vertical-Cavity SurfaceEmitting Lasers. Cambridge Univ. Press, Cambridge, UK, 1999.

3 J. Piprek. phys. stat. sol. (a), 188:905-912, 2001.

$4 \mathrm{H} . \mathrm{Li}$ and K. Iga, editors. Vertical-Cavity Surface-Emitting Laser Devices. Springer, Berlin, 2003.
5 J. Piprek, editor. Optoelectronic Devices: Advanced Simulation and Analysis. Springer, New York, 2005.

6 T. Margalith. Development of Growth and Fabrication Technology for Gallium NitrideBased Vertical-Cavity Surface-Emitting Lasers. PhD thesis, University of California at Santa Barbara, 2002.

7 A. V. Nurmikko and J. Han. Progress in blue and near-ultraviolet vertical-cavity emitters: A status report. In: H. Li and K. Iga, editors, Vertical-Cavity Surface- 
Emitting Laser Devices. Springer, Berlin, 2003.

8 J. Piprek, Y. A. Akulova, D. I. Babic, L. A Coldren, and J. E. Bowers. Appl. Phys. Lett., 72(15):1814-1816, 1998.

9 S. F. Yu. Analysis and Design of Vertical Cavity Surface Emitting Lasers. Wiley, Hoboken, 2003.

10 PICS3D by Crosslight Software Inc., Burnaby, Canada, 2005.

11 J. Piprek and S. Nakamura. IEE Proc., Part J: Optoelectron., 149:145-151, 2002.

12 I. Vurgaftman and J. R. Meyer. J. Appl. Phys., 94:3675-3691, 2003.

13 A. V. Dmitriev and A. L. Oruzheinikov. J. Appl. Phys., 86:3241-3246, 1999.

14 J. Piprek, T. Katona, S. P. DenBaars, and S. Li. 3D simulation and analysis of UV AlGaN/GaN LEDs. In: Light-Emitting Diodes: Research, Manufacturing and Applications VII, volume 5366, SPIE, Bellingham, 2004.

15 J. Piprek. Semiconductor Optoelectronic Devices: Introduction to Physics and Simulation. Academic Press, San Diego, 2003.

16 J. Piprek and S. Li. GaN-based lightemitting diode. In: J. Piprek, editor, Optoelectronic Devices: Advanced Simulation and Analysis. Springer, New York, 2005.

17 S.-H. Wei and A. Zunger. Appl. Phys Lett., 69:2719-2721, 1996

18 A. C. Abare. Growth and Fabrication of Nitride-Based Distributed Feedback Laser Diodes. PhD thesis, University of California at Santa Barbara, 2000.

19 C. G. Van de Walle and J. Neugebauer. Nature, 423:626-628, 2003

20 W. Götz, N. M. Johnson, J. Walker, D. P. Bour, and R. A. Street. Appl. Phys. Lett., 68:667-669, 1996

21 K. H. Ploog and O. Brandt. J. Vac. Sci. Technol., A16:1609-1614, 1998.

22 S. Nakamura, S. Pearton, and G. Fasol. The Blue Laser Diode. Springer-Verlag, Berlin, 2000.

23 D. M. Caughey and R. E. Thomas. Proc. IEEE, 55:2192-2193, 1967.

24 F. Schwierz. Solid State Electron., 49:889895, 2005.
25 D. K. Gaskill, L. B. Rowland, and K. Doverspike. Electrical transport properties of AlN, GaN, and AlGaN. In: J. H. Edgar, editor, Properties of GroupIII Nitrides, pages 101-116. IEE/INSPEC, London, 1994

26 T. T. Mnatsakanov, M. E. Levinshtein, L. I Pomortseva, S. N. Yurkov, G. S. Simin, and M. A. Khan. Solid State Electron. 47:111-115, 2003

27 S. L. Chuang and C. S. Chang. Phys. Rev. B, 54:2491- 2504, 1996.

28 S. L. Chuang and C. S. Chang. Semicond. Sci. Technol., 12:252-263, 1997.

29 M. D. McCluskey, C. G. Van de Walle, L. T. Romano, B. S. Krusor, and N. M. Johnson. J. Appl. Phys., 93:4340-4342, 2003.

30 C. Wetzel, T. Takeuchi, S. Yamaguchi, H. Katoh, H. Amano, and I. Akasaki. Appl. Phys. Lett., 73:1994-1996, 1998.

31 V. Fiorentini, F. Bernardini, and O. Ambacher. Appl. Phys. Lett., 80:1204-1206, 2002.

32 J. Piprek, R. Farrell, S. DenBaars, and S. Nakamura. IEEE Photon. Technol. Lett. 18:7-9, 2006.

33 S. F. Chichibu, A. C. Abare, M. S. Minsky, S. Keller, S. B. Fleischer, J. E. Bowers, E. Hu, U. K. Mishra, L. A. Coldren, and S. P. DenBaars. Appl. Phys. Lett., 73:20062008, 1998.

34 F. Renner, P. Kiesel, G. H. Döhler, M. Kneissl, C. G. Van de Walle, and N. M. Johnson. Appl. Phys. Lett., 81:490-492, 2002.

35 H. Zhang, E. J. Miller, E. T. Yu C. Poblenz, and J. S. Speck. Appl. Phys. Lett., 84:4644-4646, 2004.

36 J. P. Ibbetson, P. T. Fini, K. D. Ness, S. P. DenBaars, J. S. Speck, and U. K. Mishra. Appl. Phys. Lett., 77:250-252, 2000.

37 I. H. Brown, I. A. Pope, P. M. Smowton, P. Blood, J. D. Thomson, W. W. Chow, D. P. Bour, and M. Kneissl. Appl. Phys. Lett., 86:131108, 2005.

38 W. W. Chow and S. W. Koch. Semiconductor-Laser Fundamentals. Springer-Verlag, Berlin, 1999.

39 S. L. Chuang. IEEE J. Quantum Electron., 32(10):1791-1799, 1996. 
40 S. H. Park and S. L. Chuang. Appl. Phys. Lett., 72:287-289, 1998.

41 P. Bienstman, R. R. Baets, J. Vukusic, A. Larsson, M.J. Noble, M. Brunner, K. Gulden, P. Debernardi, L. Fratta, G.P. Bava, H. Wenzel, B. Klein, O. Conradi, R. Pregla, S.A. Riyopoulos, J.-F.P. Seurin, and S. L. Chuang. IEEE J. Quantum Electron., 37:1618 -1631, 2001.
42 G.R. Hadley, K.L. Lear, M.E. Warren, K.D. Choquette, J.W. Scott, and S.W. Corzine. IEEE J. Quantum Electron., 32:607-616, 1996.

43 G. M. Laws, E. C. Larkins, I. Harrison, C. Molloy, and D. Somerford. J. Appl. Phys., 89:1108-1115, 2001.

44 R. F. Kazarinov and M. R. Pinto. IEEE J. Quantum Electron., 30:49-53, 1994. 


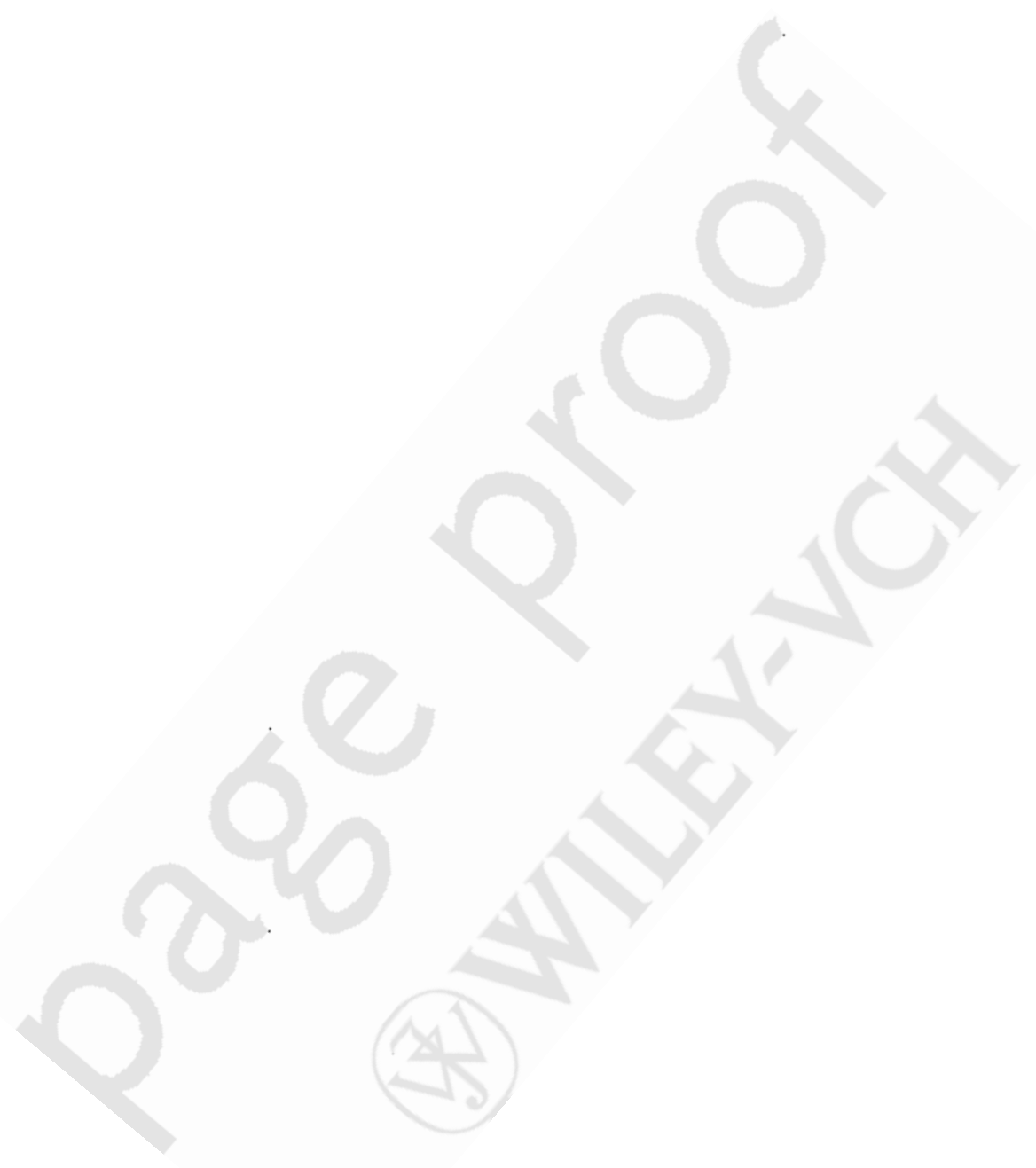

